\title{
Rapid recovery of acute transverse myelitis treated with steroids
}

\author{
P.G.E. Kennedy and A.I. Weir \\ Glasgow University Departments of Neurology and Neurophysiology, Institute of Neurological Sciences, \\ Southern General Hospital, 1345 Govan Road, Glasgow G51 4TF, UK.
}

\begin{abstract}
Summary: A case of acute transverse myelitis is described in which steroid therapy was followed by a remarkably rapid clinical and electrophysiological recovery. A possible explanation is proposed.
\end{abstract}

\section{Introduction}

An acute transverse myelitis syndrome may occur by itself or in the context of a more generalized central nervous system (CNS) disturbance such as acute disseminated encephalomyelitis (ADEM). The latter entity produces widespread demyelinating lesions throughout the CNS and may represent one extreme end of a spectrum of demyelinating disease in which multiple sclerosis is also included. The pathophysiology of the paralysis caused by acute transverse myelitis is therefore of considerable interest. A recent case of ours prompted us to consider how such a mechanism might occur.

\section{Case report}

An 18 year old man developed a flu-like illness with fever, generalized malaise and muscle aches 2 weeks before admission to the Institute of Neurological Sciences. Within a few days of the onset of his symptoms he became confused and disorientated and 10 days after the onset his legs, and to a lesser extent his arms, became weak. He also experienced thoracic and cervical pain. A marked deterioration in his leg weakness and a sudden inability to pass urine prompted his transfer to the Institute of Neurological Sciences. There were no disturbances of vision or speech. He had not been abroad recently and there was no history of exposure to individuals with infectious disease. He worked as an auxiliary nurse and there was no history of medication or drug abuse.

On examination he looked ill and was febrile

Correspondence: Professor P.G.E. Kennedy, M.D., Ph.D., M.R.C.P.

Accepted: 8 December 1987 (temperature $38.5^{\circ} \mathrm{C}$ ). General examination was unremarkable. His pulse was 80 per minute and regular and the blood pressure was $140 / 60 \mathrm{~mm} \mathrm{Hg}$. On neurological examination he was noted to be alert and orientated but very irritable. There was mild neck stiffness. Both optic discs were slightly swollen but visual acuity was $6 / 6$ bilaterally and there was no field defect. There was neither facial weakness nor bulbar involvement. In the motor system tone was slightly increased in the legs, and there was mild distal weakness in the upper limbs but severe Grade 2 (MRC scale) symmetrical weakness in both legs. Reflexes were present in the upper limbs but absent in the lower limbs and both plantar responses were absent. There was a sensory level to pin prick and light touch to the T4 level anteriorly and posteriorly.

Investigations revealed a haemoglobin of $15 \mathrm{~g} / \mathrm{dl}$, a white blood cell count of $10.2 \times 10^{9} / 1$ and there were a few activated lymphocytes suggesting a viral infection. Monospot screening test for glandular fever was negative. The routine biochemistry was normal and serological screening for a wide range of viruses and other organisms was negative. The cerebrospinal fluid (CSF) was clear and colourless with a pressure of $20 \mathrm{~cm}$ of water. It contained 34 lymphocytes $/ \mathrm{mm}^{3}$ with no organisms seen. CSF protein was $0.8 \mathrm{~g} / 1$ with a sugar of $4.0 \mathrm{mmol} / \mathrm{l}$. Oligoclonal bands were not detected. Within a few days the CSF was normal. Electroencephalogram (EEG) showed rhythmic delta activity of high amplitude bifrontally and centrally. Chest X-ray was normal as was a computed tomographic brain scan. A magnetic resonance imaging scan showed small plaques of long T2 signal in the left superior cerebellar peduncle and in the right mid portion of the pons, thought to be either oedema or demyelination. A full length myelogram was

(C) The Fellowship of Postgraduate Medicine, 1988 
normal. Visual evoked responses were normal. Peripheral nerve conduction studies in the common peroneal and tibial nerves (motor) and sural sensory potentials were normal. F-waves in the common peroneal and $\mathrm{H}$ reflexes in the tibial nerves were normal. Concentric needle electromyography showed a profound loss of voluntary motor unit potentials on maximal contraction in the absence of signs of denervation. Somatosensory evoked potentials (SEP) from stimulation of the median nerves were within the normal range bilaterally for our laboratory. SEP from stimulation of the tibial nerves were absent bilaterally. He was treated with dexamethasone $4 \mathrm{mg}$ t.d.s. at a time when his weakness was maximal and 24 hours later the power in his legs showed a remarkable improvement to Grade 4 and leg reflexes were easily obtained. The lower limb SEP were repeated at this time and showed total recovery of normal latency and amplitude responses. Subsequent improvement was then rapid, with bladder function recovering 24 hours later. On discharge from hospital 12 days later he had only mild weakness of hip flexion and ankle dorsiflexion bilaterally.

\section{Discussion}

The clinical picture and investigation strongly suggested a diagnosis of acute disseminated encephalomyelitis (ADEM) with an acute transverse myelitic component and there was clinical and radiographic evidence of several discrete lesions. The clinical and electrophysiological improvement which occurred within 24 hours of the commencement of steroid therapy was remarkable. To associate this improvement with the therapy is attractive, as the natural history of ADEM is associated with recovery over 2-6 weeks in those who recover fully ${ }^{1}$ rather than a few hours.

Evoked potentials have been studied in ADEM

\section{References}

1. Miller, H., Stanton, J. \& Gibbons, J. Parainfectious encephalomyelitis and related syndromes. $Q \mathrm{~J}$ Med 1956, 25: 427.

2. Wulff, C.H. Evoked potentials in acute transverse myelopathy. Dan Med Bull 1985, 32: 282-287.

3. Matthews, W.B. \& Small, D.G. Serial recording of visual and somatosensory evoked potentials in multiple sclerosis. J Neurol Sci 1979, 40: 11-21. previously in a group of 9 patients investigated 325 weeks after onset. ${ }^{2}$ Whilst the visual, brainstem and upper limb SEP were abnormal in all but one case, the lower limb SEP were normal in six cases. Abnormalities were either a mild delay of the cortical response or its absence. The author concluded that the findings were attributable to oedema and necrosis in the central nervous system rather than demyelination because the latter would tend to produce substantial and permanent delay as is seen in multiple sclerosis ${ }^{3}$ rather than the absent responses which in some cases recovered.

In our case the rapid clinical and electrophysiological improvement over a period of 24 hours gives some clues also as to the pathophysiology. Resolution of oedema is a well known glucocorticoid action which may be relevant here although there was in our case no radiological evidence of a macroscopically swollen spinal cord. Remyelination is a process that takes much longer than 24 hours, being more in keeping with the reports of resolution over 2-6 weeks. The most attractive pathophysiological hypothesis is of the resolution of temporary conduction block. Acute conduction block has been demonstrated experimentally by the intraneural injection of antiserum in experimental animals. This produced minimal structural alterations at the time of complete physiological block. ${ }^{4}$ Conduction block is also seen electrophysiologically in the Guillain-Barré polyneuropathy and it is relevant that in some patients with this condition a rapid recovery of the time course of our own patients may be associated with plasma exchange therapy. ${ }^{5}$

This case demonstrates the rapid resolution of afferent and efferent conduction block in the central nervous system associated with corticosteroid therapy in a case of ADEM. It underlines the importance of early diagnosis and treatment and the usefulness of evoked potentials to monitor changes in conduction in the central nervous system.

4. Sumner, A.J., Saida, K., Saida, T., Silberberg, D.H. \& Ashbury, A.K. Acute conduction block associated with experimental antiserum-mediated demyelination of peripheral nerve. Ann Neurol 1982, 11: 469-477.

5. Guillain-Barré Syndrome Study Group. Plasmapheresis and acute Guillain-Barré syndrome. Neurology 1985, 35: 1096-1104. 